\title{
QUANTIFICAÇÃO DE MICÉLIO EXTRARRADICULAR DE FUNGOS MICORRÍZICOS ARBUSCULARES EM PLANTAS CÍTRICAS. II. COMPARAÇÃO ENTRE DIFERENTES ESPÉCIES CÍTRICASE ENDÓFITOS ${ }^{(1)}$
}

\author{
R. MELLONI ${ }^{(2)} \&$ E. J . B. N. CARDOSO(3)
}

\begin{abstract}
RESUMO
Visando verificar a influência da utilização de diferentes métodos de quantificação de micélio extrarradicular ativo (MEA) e micélio extrarradicular total (MET) de fungos micorrízicos arbusculares (FMAs) e de diferentes hospedeiros nesta si mbiose, foi realizado um experimento em casa de vegetação, com delineamento inteiramente casualizado e esquema fatorial: 3 espécies de FMAs (Glomus intraradices, G. etunicatum e G. clarum) e um controle sem F MA $\times 6$ doses de $P\left(0,50,100,150,200\right.$ e $250 \mathrm{mg} \mathrm{kg}^{-1}$ de substrato) $\times 2$ plantas cítricas: tangerina cleópatra (Citrus reshni) e laranja caipira (Citrus sinensis), com 5 repetições por tratamento. Seis meses após o transplantio e inoculação das mudas, determinaram-se altura e diâmetro do caule, colheram-se as plantas e avaliaram-se matéria seca e nutrientes da parte aérea, porcentagem de colonização radicular e comprimento de MEA e MET pelos métodos de fluorescência induzida com diacetato de fluoresceína (FDA) e redução do iodonitrotetrazólio (INT). As doses crescentes de P proporcionaram redução da porcentagem de colonização radicular nos dois porta-enxertos estudados e aumentos nas características altura, diâmetro, matéria seca da parte aérea e quantidade total absorvida de macro e micronutrientes por ambos os portaenxertos. Houve correlação negativa entre comprimento de MEA e quantidade total absorvida de macronutrientes ( $\mathrm{P}, \mathrm{N}, \mathrm{Ca}$ e Mg) por laranja cai pira e correlação positiva entre comprimento de MET e quantidade absorvida por tangerina cleópatra, sugerindo ser esta última mais micotrófica que a anterior, além de haver, possivel mente, outros mecani smos de absorção de nutrientes que não pelo MEA, cujos resultados variaram em função do método empregado.
\end{abstract}

Termos de indexação: corante, FDA, INT.

(1) Parte da Tese de Mestrado do primeiro autor. Trabalho realizado com auxílio da FAPESP. Recebido para publicação em junho de 1997 e aprovado em setembro de 1998.

(2) Mestre em Agronomia pela Escola Superior de Agricultura Luiz de Queiroz - ESALQ/USP. Av. Pádua Dias 11, Caixa Postal 9, CEP 13418-900 Piracicaba (SP). Bolsista do CNPq.

(3) Professora Titular do Departamento de Solos e Nutrição de Plantas, ESALQ/USP. Bolsista do CNPq. 


\title{
SUMMARY: QUANTIFICATION OF EXTRARADICULAR MYCELIUM OF ARBUSCULAR MYCORRHIZAL FUNGI ON CITRUS. II. COMPARISONS BETWEEN DIFFERENT CITRUS AND ENDOPHYTE SPECIES
}

\begin{abstract}
Different methods for quantification of active extraradicular mycelium (AEM) and total extraradicular mycelium (TEM) of arbuscular mycorrhizal fungi (AMF) wereexamined by conducting an experiment under greenhouse conditions, with a completely randomized factorial design and five replicates per treatment. The experiment was arranged in a $4 \times 6 \times 2$ design and thefactors were 3 species of AMF (Glomus intraradices, G. etunicatum and G. darum) and one control without AMF $\times 6$ levels of $P(0,50,100,150,200$ and $250 \mathrm{mg} \mathrm{kg}^{-1}$ ) $\times 2$ citrus species (Citrus reshni and Citrus sinensis). Six months after transplanting, height and diameter were evaluated and the plants were harvested. Shoot dry weight, total shoot macronutrients, percentage of root infection and thelength of $A E M$ and TEM by the fluoresce $n$ diacetate (FDA) and the iodonitrotetrazolium reduction (INT) methods wereal so evaluated. Increasing levels of $P$ promoted decreases in percentage of root infection and increases in plant height, diameter, dry weight and macro and mi cronutrients absorbed by both citrus species. There was a negative correlation between AEM and total macronutrients absorbed $(\mathrm{P}, \mathrm{N}, \mathrm{Ca}$ and $\mathrm{Mg}$ ) by Citrus sinensis and a positive correlation between TEM and those macronutrients by $\mathrm{C}$. reshni indicating that thelatter host may be moremycotrophic than theformer and, possi bly, that thereareother mechanisms of nutrient absorption rather than by $A E M$, with results varying in relation to the method used.
\end{abstract}

Index terms: FDA, INT, vital stain.

\section{INTRODUÇÃO}

Plantas com sistema radicular profundo e pobre em pêlos absorventes, como citros, são altamente micotróficas e dependentes de fungos micorrízicos arbusculares (F MAs) para a absor ção de nutrientes (principalmente P) e seu desenvol vimento (Fonseca et al., 1994). O P éo nutriente mais importantenesse complexo nutricional pelo fato de estar pouco disponível, apresentar baixa mobilidade e estar em baixa concentração no solo. Então, qualquer tipo de manejo que aumente a absorção ea utilização desse nutriente seria de extrema importância para a agricultura, resultando em maior economia de fertilizantes fosfatados, fonte de insumo nãorenovável.

A significativa absorção de $\mathrm{P}$ por plantas micorrizadas tem sido atribuída à maior exploração do solo e à maior capacidade das hifas extrarradiculares em absorver $\mathrm{P}$ em baixa concentração (Silveira \& Cardoso, 1990; Tobar et al ., 1994; Sena et al., 1997). Segundo Cardoso et al. (1986), a utilização de FMAs de grande eficiência pode promover aumentos de até $5.070 \%$ no $\mathrm{P}$ acumulado na parte aérea dos hospedeiros.

$O$ efeito de $P$ do substrato sobre a mi corrização ocorre na fase de crescimento rápido com o desenvolvimento de micélio extrarradicular, múltiplas infecções e extensivo desenvolvimento interno de cada espécie de FMA (Abbott \& Robson, 1985; Lambais \& Cardoso, 1988). Baixas concentrações de P na planta já foram relacionadas com o maior desenvolvimento de micélio extrarradicular (Miranda \& Harris, 1994a, b), assim como diferentes fontes deP in vitro (Silva et al., 1991) e espécies de F M As ( akobsen et al., 1992). Portanto, o desenvolvimento micelial constitui importante fator na determinação da capacidade do FMA em aumentar o crescimento vegetativo, mas a sua quantificação sempre foi associada a dificuldades técnicas, principalmente na diferenciação entre fungos MAs e os não-micorrízicos, e ao uso de métodos trabal hosos de extração eaval iação (Sylvia, 1992; Vilariño et al., 1993; Cardoso Filho, 1994).

Segundo Sylvia (1988), independentemente da importância do mi célio extrarradicular na absorção e translocação de nutrientes, muitos pesquisadores têm-se dedicado apenas à quantificação do comprimento de mi célio total de FMAs no solo, sem distinção de micélio ativo e inativo. Uma vez que a absorção de P é um processo ativo (Beever \& Burns, 1980; Schubert et al., 1987), a quantificação de micélio ativo seria de extrema importância. Há dúvidas quanto ao método mais apropriado, visto que diferentes técnicas podem fornecer resultados discrepantes (Hamel et al., 1990; Melloni \& Cardoso, 1998). Os métodos enzi máticos têm sido largamente utilizados nesta avaliação, destacando-se os de fluorescência induzida com a hidrólise do diacetato de fluoresceína-FDA (Hamel et al., 1990; Sylvia, 1992; Gomes, 1997; Nogueira, 1997), indicando a presença de esterases, e o de redução do iodonitrotetrazólio-INT (Sylvia, 1992; Cardoso Filho, 1994; Kabir et al., 1997), para desidrogenases, presentes em organismos vivos. 
Os objetivos do presentetrabal ho foram verificar a influência de utilização desses dois métodos enzimáticos de quantificação de micélio extrarradicular ativo (MEA) etotal (MET) deFMAs em estudos nutricionais de dois porta-enxertos de citros (laranja caipira e tangerina cleópatra) micorrizados.

\section{MATERIAL E MÉTODOS}

Desenvolveu-se um experimento em casa de vegetação com delineamento inteiramente ao acaso e esquema fatorial 6 doses de $P(0,50,100,150,200$ e $250 \mathrm{mg} \mathrm{kg}^{-1}$ de substrato) na forma de $\mathrm{KH}_{2} \mathrm{PO}_{4} \mathrm{e}$ $\mathrm{NaH}_{2} \mathrm{PO}_{4} \cdot \mathrm{H}_{2} \mathrm{O} \times 4$ níveis de inoculação [(3 espécies de FMAs (Glomus intraradices, G. etuni catum e G. clarum) eum controle (sem F MA)] x 2 porta-enxertos cítricos [(tangerina cleópatra (Citrus reshni) elaranja caipira (Citrus sinensis)], com 5 repetições, em vasos plásticos de 5 litros. O substrato foi composto de 3 partes de solo Areia Quartzosa série Paredão Vermel ho ( $\mathrm{pH}$ em $\mathrm{CaCl}_{2}$ 3,8; $11 \mathrm{~g} \mathrm{dm}^{-3}$ de M.O.; $3 \mathrm{mg} \mathrm{dm}^{-3}$ de $\mathrm{P}$ resina; 0,$2 ; 7 ; 2 ; 8$ e $28 \mathrm{mmol}_{\mathrm{c}} \mathrm{dm}^{-3}$ de $+, \mathrm{Ca}^{+2}, \mathrm{Mg}^{+2}, \mathrm{Al}+3 \mathrm{eH}++\mathrm{Al}+3$, respectivamente), para 1 parte de areia de rio peneirada e lavada, autoclavada por $2 \mathrm{~h}$ a $121^{\circ} \mathrm{C}$, corrigido e adubado conformeAntunes \& Cardoso (1991). O substrato dos vasos foi infestado com propágulos de FMAs, utilizando-se $20 \mathrm{~g}$ de solo rizosféricoe raízes de milho Piranão micorrizado, antes do transplantio de uma muda de tangerina cl eópatra ou laranja cai pira por vaso, obtida após 3 meses de crescimento em caixas de areia autoclavada. Para os vasos de controle, utilizou-se a mesma quantidade de solo rizosférico de plantas não inoculadas.

Seis meses após o transplantio, determinaramsealtura e diâmetro do caule, col heram-se as plantas eavaliaram-se: (a) matéria seca da parteaérea, após peso constanteem estufa a $60^{\circ} \mathrm{C}$; (b) macronutrientes ( $\mathrm{N}, \mathrm{P}, \mathrm{K}, \mathrm{Ca}, \mathrm{Mg})$ e micronutrientes (Cu, Fe e Mn) da parte aérea, segundo método descrito em Sarruge \& Haag (1974); (c) porcentagem de colonização radicular (Phillips \& Hayman, 1970; Giovanetti \& Mosse, 1980; Bethlenfalvay \& Ames, 1987) e (d) comprimentos de micélio extrarradicular ativo (MEA) emi célio extrarradicular total (MET) deFMAs pel os métodos defluorescência induzida com hidrólise dediacetato de fluoresceína-FDA (Hamel et al., 1990; Sylvia, 1992) ede redução de iodonitrotetrazólio-INT (Benefield et al., 1977; Cardoso Filho, 1994), após extração de micélio por meio do método predefinido por Melloni \& Cardoso (1998).

Os dados foram submetidos à análise de variância, utilizando-se o nível de significância de 0,05 para o teste $F$. Val ores relativos à porcentagem de col onização radicular foram transformados para arc sen (x/100)1/2, sendo as comparações de médias (Tukey) e as equações de regressão feitas por meio do programa estatístico SANEST (Zonta et al., 1984).

\section{RESULTADOS E DISCUSSÃO}

\section{Altura, diâmetro de caule, matéria seca da parte aérea e porcentagem de colonização radicular dos porta-enxertos}

Verificaram-se efeitos das interações portaenxerto-P e P-F MAs nas variáveis altura de planta e matéria seca da parte aérea. O comportamento da variável matéria seca da parte aérea em função das doses de $\mathrm{P}$ pôdeser representado por equaçãolinear para o porta-enxerto tangerina cleópatra ( $\left.y=0,019 x+1,0 \quad R^{2}=0,90 * *\right)$ e quadrática para laranja caipira $\left(y=-5,1 \cdot 10-5 x^{2}+0,024 x+0,83\right.$ $\mathrm{R}^{2}=0,96 *$, revelando maior resposta do primeiro aoP. Houve correl ação al tamente significativa entre matéria seca da parte aérea e altura de plantas, concordando com os resultados obtidos por Gomes (1997). O efeito defungos micorrízicos arbusculares (FMAs) na matéria seca da parte aérea em função das doses de $\mathrm{P}$ pode ser visto no quadro 1 , onde $\mathrm{G}$. clarum e G. intraradices destacaram-se dos demais em praticamentetodas as doses de P e porta-enxertos, com médias estatisticamente superiores às de $\mathrm{G}$. etunicatum e controle sem FMA. Para médias de ambos os porta-enxertos, verificou-se efeito quadrático de doses de $P\left(y=-2,7 \cdot 10^{-5} x^{2}+0,015 x+2,55\right.$ $\left.\mathrm{R}^{2}=0,98^{* *}\right)$, com médias para laranja caipira significativamente superiores às de tangerina cleópatra quando colonizada por G. clarum ou G. intraradices (resultados não apresentados).

$O$ efeito de doses de $P$ nessas variáveis já era esperado, pel o fato de ter sido este o único nutriente limitantena referida condição experimental. Diversos autores (Lambais \& Cardoso, 1990; Souza et al ., 1991; Fonseca et al., 1994; Melloni et al., 1996a,b; Gomes, 1997; N ogueira, 1997), em estudos com micorrizas, constataram correlação positiva esignificativa entre matéria seca da parte aérea e $\mathrm{P}$ total absorvido, conforme ocorreu em ambos os porta-enxertos. Os fungos G. clarum e G. intraradices foram os FMAs que mais se destacaram nessas variáveis, provavelmente por apresentarem alta eficiência na col onização radicular de ambos os porta-enxertos (Quadro 2).

Verificou-se efeito negativo das doses de P na porcentagem de col onização radicular dos porta-enxertos para todos os fungos micorrízicos arbusculares (FMAs) utilizados. Observou-se que G. intraradices apresentou efeito linear negativo de colonização radicular com o aumento das doses de $\mathrm{P}$ para ambos os porta-enxertos $\left(y=-0,042 x+24,6 R^{2}=0,42 * *\right.$ para Iaranja cai pira e $y=-0,122 x+32,2 R^{2}=0,90 * *$ para tangerina cleópatra), enquanto $\mathrm{G}$. clarum mostrou efeito quadrático $\left(y=-5,810^{-4} x^{2}+0,092 x+32,7 R^{2}=\right.$ $0,68 * *$ para laranja caipira e $y=-7,310^{-4} x^{2}+0,11 x$ $+35,2 R^{2}=0,64 * *$ para tangerina cleópatra). Pelos resultados obtidos, este último parece ser menos sensível ao aumento das doses de $\mathrm{P}$, quando comparado ao G. intraradices, já que, mesmo em doses elevadas deP, ainda promoveu colonização dos 
Quadro 1. Matéria seca da parte aérea dos porta-enxertos (média de 10 repetições) influenciada por fungo micorrízico arbuscular e doses de fósforo e respectivas equações de regressão

\begin{tabular}{|c|c|c|c|c|c|c|c|c|}
\hline \multirow{2}{*}{$\begin{array}{c}\text { Fungo micorrízico } \\
\text { arbuscular }\end{array}$} & \multicolumn{6}{|c|}{ Doses de P (mg kg ${ }^{-1}$ de substrato) } & \multirow{2}{*}{\multicolumn{2}{|c|}{ E quação de regressão }} \\
\hline & $\mathbf{0}$ & 50 & 100 & 150 & 200 & 250 & & \\
\hline & & & & 9 & 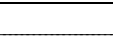 & 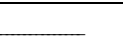 & & \\
\hline Controle & $0,17 a$ & $0,35 \mathrm{~b}$ & $1,01 \mathrm{~b}$ & $1,51 \mathrm{~b}$ & $2,91 \mathrm{~b}$ & $3,86 \mathrm{bc}$ & $\hat{\mathrm{Y}}$ & $=0,015 x-0,27 R^{2}=0,94 * *$ \\
\hline G. intraradices & $1,41 \mathrm{a}$ & $3,52 \mathrm{a}$ & $4,16 \mathrm{a}$ & $4,38 \mathrm{a}$ & $4,62 b$ & $5,26 a b$ & $\hat{\mathrm{Y}}$ & $=0,013 x+2,26 R^{2}=0,82 * *$ \\
\hline G. etunicatum & $0,19 a$ & $0,48 \mathrm{~b}$ & $1,18 \mathrm{~b}$ & $1,75 \mathrm{~b}$ & $2,84 \mathrm{~b}$ & $2,94 \mathrm{c}$ & $\hat{\mathrm{Y}}$ & $=0,012 x+0,035 R^{2}=0,97 * *$ \\
\hline G. clarum & $1,24 a$ & $4,84 \mathrm{a}$ & 3,99 a & $5,80 \mathrm{a}$ & $7,45 \mathrm{a}$ & $6,12 a$ & $\hat{\mathrm{Y}}$ & $=-1 \cdot 10^{-4} x^{2}+0,046 x+1,60 R^{2}=0,83 * *$ \\
\hline
\end{tabular}

Comparam-se letras na vertical. Médias seguidas por letras iguais não diferem entre si (Tukey a 5\%). ** significativo a $1 \%$.

Quadro 2. Colonização radicular dos porta-enxertos laranja caipira e tangerina cleópatra, colonizados por FMAs, considerando as doses crescentes de P (média de 5 repetições)

\begin{tabular}{|c|c|c|c|c|c|c|}
\hline \multirow{2}{*}{$\begin{array}{c}\text { Doses P } \\
\left(\mathrm{mg} \mathrm{kg}^{-1}\right)\end{array}$} & \multicolumn{2}{|c|}{ G. intraradices } & \multicolumn{2}{|c|}{ G. etunicatum } & \multicolumn{2}{|c|}{ G. clarum } \\
\hline & $\begin{array}{c}\text { Laranja } \\
\text { caipira }\end{array}$ & $\begin{array}{l}\text { Tangerina } \\
\text { cleópatra }\end{array}$ & $\begin{array}{c}\text { Laranja } \\
\text { caipira }\end{array}$ & $\begin{array}{l}\text { Tangerina } \\
\text { cleópatra }\end{array}$ & $\begin{array}{l}\text { Laranja } \\
\text { caipira }\end{array}$ & $\begin{array}{l}\text { Tangerina } \\
\text { cleópatra }\end{array}$ \\
\hline 0 & $14,2 \mathrm{bB}$ & $27,8 \mathrm{aA}$ & $2,0 \mathrm{aC}$ & $0,2 \mathrm{aB}$ & $35,2 \mathrm{aA}$ & $38,6 a A$ \\
\hline 50 & $19,9 a A$ & $20,4 a A$ & $2,5 \mathrm{aB}$ & $0,1 \mathrm{aB}$ & $22,5 \mathrm{aA}$ & $34,2 \mathrm{aA}$ \\
\hline 100 & $17,5 \mathrm{aB}$ & $14,7 \mathrm{aB}$ & $0,1 \mathrm{aC}$ & $0,0 \mathrm{aC}$ & $39,2 \mathrm{aA}$ & $32,6 a A$ \\
\hline 150 & $3,4 \mathrm{aB}$ & $4,6 a B$ & $0,8 \mathrm{aB}$ & $0,0 \mathrm{aC}$ & $32,0 a A$ & $31,0 a A$ \\
\hline 200 & $6,6 \mathrm{aB}$ & $0,1 \mathrm{bB}$ & $0,6 \mathrm{aC}$ & $0,0 \mathrm{aB}$ & $26,6 \mathrm{bA}$ & $39,8 a A$ \\
\hline 250 & $8,7 a A$ & $1,2 \mathrm{bAB}$ & $0,1 \mathrm{aB}$ & $0,0 \mathrm{aB}$ & $8,9 a A$ & $4,0 \mathrm{aA}$ \\
\hline
\end{tabular}

Comparam-se letras minúsculas na horizontal entre porta-enxertos para o mesmo $\mathrm{FMA}$ e letras maiúsculas entre $\mathrm{FMAs}$ do mesmo porta-enxerto. Médias seguidas de letras iguais não diferem entre si (Tukey a 5\%).

hospedeiros. O efeito negativo de doses de $\mathrm{P}$ na colonização radicular por $\mathrm{G}$. intraradices e G. clarum está de acordo com Antunes \& Cardoso (1991), Peng et al. (1993), Miranda \& Harris (1994b), Melloni et al. (1996a,b), Nagahashi et al. (1996), Gomes (1997) e Nogueira (1997), com hipóteses ligadas à diminuição dos exsudatos radiculares, interferindo no processo inicial de desenvolvimento micelial de FMAs (Graham et al., 1981; Miranda \& Harris, 1994a); ao aumento de $P$ foliar com diminuição da permeabilidaderadicular à infecção (Thomson et al ., 1986; Miranda et al., 1989); à variação do fluxo de carboi dratos da planta ao endófito (Buwal da $\&$ Goh, 1982; Siqueira et al ., 1984); ao aumento da atividade de endoquitinases e de $\beta-1,3$ endoglucanases, restringindo a colonização interna (Lambais \& Mehdy, 1993; Lambais \& Mehdy, 1995), entre outras. O FMA G. etunicatum, apesar de utilizado previamente por Antunes \& Cardoso (1991), não demonstrou capacidade de col onização radicular em nenhum dos porta-enxertos utilizados, provavelmente por causa da ineficiência enzimática ou morfológica no processo inicial de infecção radicular.

\section{Absorção de nutrientes pelos porta-enxertos}

O conteúdo de nutrientes ( $\mathrm{N}, \mathrm{P}, \mathrm{Ca}$ e $\mathrm{Mg}$ ) para tangerina deópatra, considerando as doses aplicadas de $P$, pôde ser expresso por equações lineares $\left[\mathrm{y}(\mathrm{N})=0,54 \mathrm{x}+23,26 \mathrm{R}^{2}=0,94 * * ; \mathrm{y}(\mathrm{P})=0,01 \mathrm{x}+1,19\right.$ $\mathrm{R}^{2}=0,89 * * ; \mathrm{y}(\mathrm{Ca})=0,26 \mathrm{x}+14,75 \mathrm{R}^{2}=0,89 * *$; $y(\mathrm{Mg})=0,07 x+4,0 R^{2}=0,91 * *$, enquanto para laranja cai pira verificou-seum efeito quadrático para a maioria dos nutrientes avaliados $[\mathrm{y}(\mathrm{N})=$ $-0,002 x^{2}+0,77 x+31,0 R^{2}=0,95 * ; y(P)=0,02 x+0,85$ $\mathrm{R}^{2}=0,90 * * ; \mathrm{y}(\mathrm{Ca})=-0,0008 \mathrm{x}^{2}+0,39 \mathrm{x}+17,22$ $R^{2}=0,92 * ; y(M g)=-0,0002 x^{2}+0,097 x+4,66$ $\mathrm{R}^{2}=0,91^{*}$ ]. Destacou-se maior conteúdo de $\mathrm{P}$ em tangerina cleópatra nas doses iguais e superiores a $200 \mathrm{mg} \mathrm{kg}^{-1}$ deP no substrato, o que poderia explicar o maior desenvolvimento vegetativo desse portaenxerto, quando comparado à laranja caipira nessas doses (dados não apresentados). Aumentos do conteúdo de nutrientes ( $P, K, S, C u, F e$ e Mn) em função das doses de $\mathrm{P}$ poderiam ser explicados pelo aumento da matéria seca da parte aérea das plantas (Quadro 1), reflexo, possivelmente, de três fatores: (a) exigência proporcional de nutrientes com o 
crescimento e necessidade de manter um equilíbrio nutricional interno; (b) aumento do sistema radicular das plantas em função das doses de $\mathrm{P}$, garantindo maior exploração do substrato e maior absorção de nutrientes, ou (c) maior desenvol vimento do micélio extrarradicular total, garantindo maior exploração superficial de substrato, transpondo a zona de depleção nutricional da raiz.

Quanto ao efeito deFMAs na composição mineral das plantas, houve resposta no conteúdo de N, S, Ca e Mg da parte aérea dos porta-enxertos, com G. clarum e G. intraradices novamente se destacando como os FMAs responsáveis pel os maiores valores. O conteúdo de $\mathrm{P}$ na planta relacionado com os FMAs com a apli cação de doses de P no substrato pôde ser representado por equação linear para G. intraradices ( $y=0,014 x+1,98 \quad R^{2}=0,88 * *$ ) e equação quadrática para G. clarum $\left(y=-9,4 \cdot 10^{-5} x^{2}+0,043 x+1,50\right.$ $\left.\mathrm{R}^{2}=0,83^{* *}\right)$, com um pico de absorção para este último na dose calculada de $229 \mathrm{mg} \mathrm{kg}^{-1}$ de $\mathrm{P}$ no substrato. Enquanto G. intraradices destacou-se pelo maior conteúdo de P comparado ao G. clarum, G. etunicatum não diferiu do controle sem FMA em nenhuma dose aplicada.

Observou-se comportamento linear positivo de conteúdo de $\mathrm{P}$ e matéria seca da parte aérea com o aumento das doses de $P$ para os fungos $G$. intraradices, G. etunicatum e controle sem FMA. Entreanto, G. clarum apresentou efeito quadrático, com diminuição dos val ores dessas duas variáveis a partir da dose teórica de $229 \mathrm{mg} \mathrm{kg}^{-1}$ de $\mathrm{P}$ no substrato. E mbora esse fungo, em doses menores de $\mathrm{P}$, promova associações simbióticas mais eficientes que os demais FMAs, possivel mente em doses superiores às utilizadas poderia ocorrer depressão no crescimento vegetativo em ambos os porta-enxertos, fenômeno demonstrado por diversos autores que utilizaram outras espécies cítricas e outros FMAs (Antunes \& Cardoso, 1991; Peng et al., 1993; Gomes, 1997).

\section{Micélio extrarradicular ativo (MEA) e total (MET) de F MAs}

Para o método FDA, verificou-se efeito quadrático do comprimento de MEA de acordo com as doses de $P$, enquanto, para o método INT, o efeito pôde ser representado por equaçãolinear, em Iaranja caipira eem tangerina cleópatra (Figura 1 ). A tendência das curvas deambos os métodos no mesmo porta-enxerto foi semel hante, com dimi nuição do comprimento de MEA para Iaranja cai pira eaumento para tangerina cleópatra até a dose $200 \mathrm{mg} \mathrm{kg}^{-1}$ de P no substrato. Como ambos os métodos são enzi máticos e cada um atua em complexos enzimáticos diferentes, resultados controvertidos poderiam ser esperados, como de fato ocorreu, concordando com Hamel et al . (1990). Segundo Beever \& Burns (1980), altas doses de $\mathrm{P}$ podem inibir o ciclo de Krebs e, como o INT é também reduzido por enzimas presentes neste cido, o método apresentaria maior sensibilidade e tenderia a indicar menor quantidade de MEA, quando comparado ao método FDA nas mesmas doses de $P$.

Não houve efeito significativo de FMAs na variável comprimento de MEA, mas verificou-se efeito positivo do aumento das doses de $\mathrm{P}$, quando associados ao porta-enxerto tangerina cleópatra (Figura 2). Apesar deser a susceti bilidade da planta à micorrização controlada geneticamente, a diferença de comportamento verificada entre os porta-enxertos se deve, provavelmente, a fatores morfológicos e, ou, fisiológicos, ligados à facilidade

$$
\begin{aligned}
& \hat{Y}(\text { INT })=-2,1 \cdot 10^{-3} x+0,60 R^{2}=0,72^{* *} \\
& \hat{Y}(\text { FDA })=1,1 \cdot 10^{-5} x^{2}-5,1 \cdot 10^{-3} x+0,73 \quad R^{2}=0,97 * *
\end{aligned}
$$

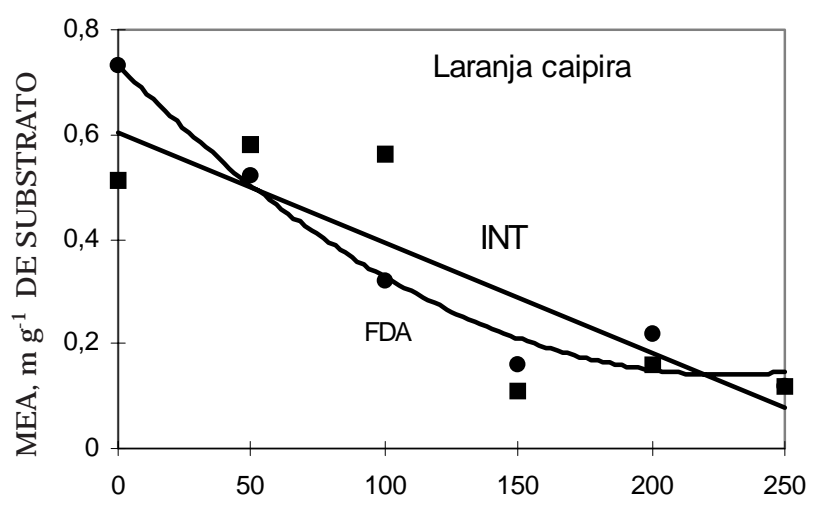

$$
\begin{aligned}
& \hat{Y}(\text { INT })=5 \cdot 10^{-4} x+0,07 \quad R^{2}=0,38^{\text {ns }} \\
& \hat{Y}(\text { FDA })=-1,3 \cdot 10^{-5} x^{2}+4,1 \cdot 10^{-3} x-0,06 \quad R^{2}=0,27^{* *}
\end{aligned}
$$

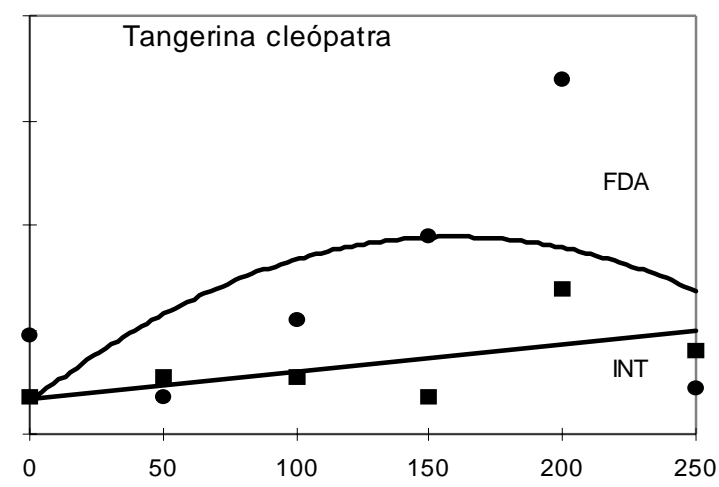

DOSES DE P, $\mathrm{mg} \mathrm{kg}^{-1}$ DE SUBSTRATO

Figura 1. Comprimento de mi célio extrarradicular ativo (MEA) de fungos micorrízi cos arbusculares para laranja cai pira e tangerina cleópatra, pelos métodos de fluorescência induzida (FDA) e redução de iodonitrotetrazólio (INT), considerando as doses aplicadas de P (média de 20 repetições). 
de absorção de $\mathrm{P}$ e outros nutrientes pela raiz; à exigência diferenciada de $\mathrm{P}$ pelo hospedeiro, à morfologia radicular ligada à penetração do fungo; à produção e liberação de exsudatos radiculares responsáveis pela atração e formação da comuni dade biológica na micorrizosfera, eà espécie ou ao isolado de FMA utilizado, cujo grau de compatibilidade fungo-planta parece estar ligado a mecanismos de reconhecimento entre os simbiontes (Silveira, 1992).

Foram encontrados valores consideráveis de comprimento de MEA em substratos não infestados com propágul os de F MAs (controle), evidenciando a existência de fungos sapróbios, oriundos provavelmente do inóculo original ou de contaminação natural, já que o substrato foi previamente autoclavado. Hifas de fungos nãomicorrízicos não são facilmente distinguíveis daquelas produzidas pelos FMAs, por não haver diferenças morfológicas ou de col oração expressivas entre ambas (Abbott et al., 1984; Abbott \& Robson, 1985; Sylvia, 1992).

Apesar dessa dificuldade de diferenciação, o comprimento de MEA de fungos sapróbios (tentativamente identificados pela maior espessura e coloração mais escura) foi superior, embora não estatisticamente diferente, em substrato-controle (principalmente em tangerina cl eópatra) do que em substrato infestado com FMAs, sugerindo inibição de desenvolvimento micelial dos primeiros na presença dos F MAs e impossibilitando a subtração dos val ores, como sugerido por Sylvia (1992) e Kabir et al. (1996) para micélio extrarradicular. I sto vem comprovar quea micorrizosfera difere profundamente da rizosfera de plantas não micorrizadas, como já discutido por Silveira (1992), e quea única maneira realmente confiável para identificar espécies de FMAs seria por meio de métodos como imunofluorescência (Kough et al., 1983; Wilson et al., 1983) ou moleculares (Lanfranco et al., 1993; Di Bonito et al., 1995; Lanfranco et al., 1995). Para todos os FMAs de laranja cai pira, observou-se redução acentuada de comprimento de MEA com o aumento das doses de $P$, cuja causa pode estar associada aos nutrientes acrescentados quando da adubação com $\mathrm{KH}_{2} \mathrm{PO}_{4}$, principalmente $\mathrm{OK}^{+}$. Essenutriente exerceria efeito inibitório no crescimento micelial pelo fato de a planta micorrizada apresentar maior absorção e concentração intracelular de P (e de K), podendo atingir níveis tóxicos ao fungo (Silva et al., 1991).

O porta-enxertolaranja caipira promoveu maior comprimento de MEA, principalmente em doses iguais ou inferiores a $100 \mathrm{mg} \mathrm{kg}^{-1}$ de $\mathrm{P}$, quando comparado ao tangerina deópatra, independen-temente do método de avaliação empregado (Figura 1), bem como do FMA (Figura 2), sugerindo um cuidado adicional em estudos interligados, considerando a grande influência do hospedeiro nessa variável.

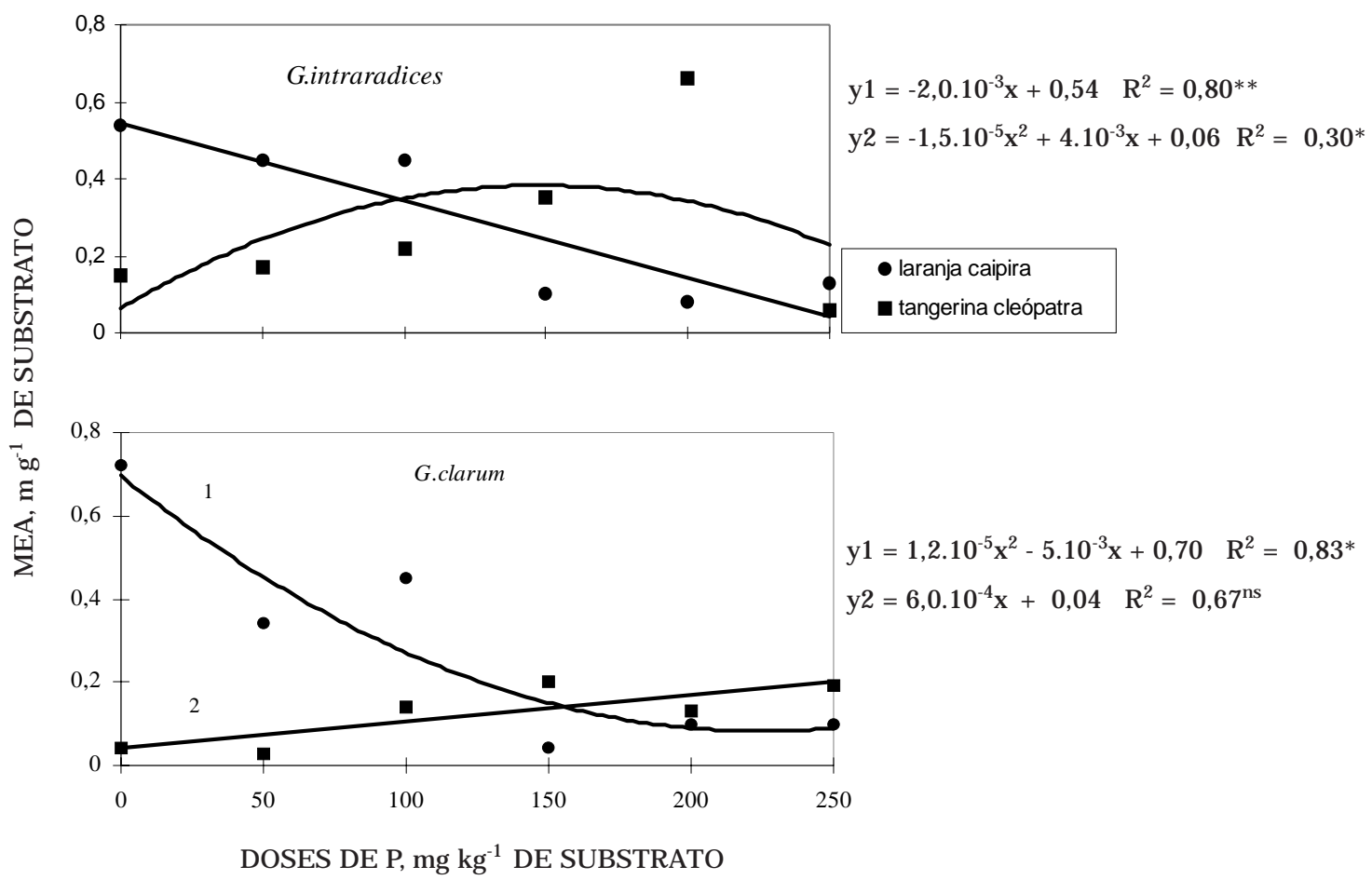

Figura 2. Comprimento de micélio extrarradicular ativo (MEA) de G. intraradices e G. clarum, nos portaenxertos laranja cai pi ra e tangeri na cleópatra, consi derando as doses aplicadas de P e métodos F DA e INT (média de 10 repetições). 
Correlações negativas significativas $(r=-0,96 * * e$ $r=-0,71 *$ ) foram encontradas entre comprimento de MEA deFMAs (métodos FDA eINT, respectivamente) eP total absorvido por Iaranja cai pira, contrariando resultados obtidos em experimentos que utilizaram limão-cravo (Melloni et al., 1996b) etrevo (Schubert et al., 1987). Esta poderia ser uma resposta da própria planta ao desenvol vimento micelial deFMA, a qual estaria absorvendo $P$ pela raiz, independentemente da presença do fungo, não havendo necessidade de despender energia para que se mantivessem as hifas ativas do fungo, responsáveis pela absorção do nutriente.

Verificou-se o oposto para tangerina cleópatra com correlação positiva e significativa $\left(r=0,68^{*}\right)$ entre comprimento de MEA (método INT) e P total absorvido, concordando com os autores supracitados e revelando que a absorção de $\mathrm{P}$ ocorreu realmente pelo MEA, provavel mente num processo ativo com dispêndio de energia pelo hospedeiro (Beever \& Burns, 1980; Cooper \& Tinker, 1981; Sylvia, 1992; Kabir et al., 1997; Bago et al., 1998).

Quanto ao MET de FMAs, não se verificou diferença estatística significativa em doses iguais e inferiores a $150 \mathrm{mg} \mathrm{kg}^{-1}$ de $\mathrm{P}$ para ambos os portaenxertos. No entanto, em doses superiores, a tangerina cleópatra superou significativamente a Iaranja cai pira, com comportamentos representados por equação linear para o primeiro porta-enxerto $\left(y=0,014 x+7,11 \quad R^{2}=0,56^{* *}\right)$ e sem ajuste significativo para o segundo. Essa observação contrariou os resultados obtidos por Abbott \& Robson (1985), Miller et al. (1987), Miranda et al. (1989), Miranda \& Harris (1994a,b), dentre outros, que encontraram redução do crescimento micelial extrarradicular com o aumento da concentração de P na planta, e assemel hou-se aos obtidos por Crush (1976) em leguminosas, Cardoso-Filho (1994) em milho, Melloni et al. (1996b) em limão-cravo e Gomes (1997) em espécies cítricas.

Abbott et al. (1984) discutiram que o P poderia atuar ainda não diretamente no crescimento micelial, mas no aumento da densidade radicular, reduzindo a distância que o micélio deveria crescer para interceptar a raiz do hospedei ro. Tais resultados revelam que plantas com maior quantidade de $\mathrm{P}$ absorvida e acumulada (aquelas mais propensas à depressão de crescimento quando micorrizadas) poderiam destinar mais fotoassimilados para a formação de maior comprimento de MET, enquanto, proporcionalmente, pequena parte estaria ativa e envol vida no processo de absorção do nutriente, como verificado na figura 1.

Para laranja caipira, encontraram-se correlações positivas e significativas ( $r=0,78^{*}$ e $r=0,83^{*}$, respectivamente) entre comprimento de MEA de G. intraradices e G. etunicatum e porcentagem de colonização radicular. Isto indica, provavelmente, uma extensão das estruturas fúngicas ativas presentes no interior da raiz altamente colonizada, corroborando com Sylvia (1988), apesar da baixa eficiência do último FMA. Teoricamente, tal comportamento era esperado, visto que, havendo maior comprimento de MEA explorando o solo, deveria existir conjuntamente maior quantidade de estruturas internas como mi célio e ar búscul os para que se efetuassem as trocas nutricionais entre os simbiontes. Para o porta-enxerto tangerina cleópatra e para o fungo $\mathrm{G}$. clarum, não foram encontradas correlações significativas para essas variáveis.

Além do P total absorvido, discutidoanteriormente, observou-se correlação negativa significativa entre totais absorvidos de N, Ca, Mg e comprimento de MEA para Iaranja caipira, enquanto para tangerina deópatra encontrou-secorrelação positiva significativa entre os mesmos macronutrientes e comprimento de MET.Tais correlações indicam que podem existir outros mecanismos de absorção e translocação de nutrientes que não pel o MEA, como já discutido por Schubert et al. (1987) e Miranda et al. (1989).

Os dados revelam que: (a) a absorção desses nutrientes poderia não ser promovida exclusivamente pelo MEA ou (b) a alta quantidade absorvida de nutrientes pela raiz ou talvez pelo micélio extrarradicular inativo (compreendido no MET) promoveria um efeito "feed-back" negativo, reduzindo o comprimento de MEA e evitando o desvio desnecessário de fotoassimilados do hospedei roao F MA. Miranda et al. (1989) eJ akobsen et al. (1994) discutiram, neste sentido, queuma alta quantidade absorvida de $\mathrm{P}$ pelo MEA poderia diminuir a absorção radicular, ou a presença dessa absorção radicular poderia impedir o funcionamento ótimo do MEA pela competição por $P$ ou interações com microrganismos da rizosfera. A existência de correlações positivas entre $P$ absorvido e MEA e entre macronutrientes absorvidos ( $\mathrm{P}, \mathrm{N}, \mathrm{Ca}$ e Mg) e MET somente em tangerina cl eópatra indica ser ele o porta-enxerto com maior dependência mi corrízica à absorção de nutrientes, ao contrário do portaenxertolaranja caipira, que seria capaz de absorvêlos por suas próprias raízes. Segundo Oliveira \& J esus (1987), tangerina cl eópatra dispõe de um sistema radicular com dominância apical e uma baixa relação de massa entre raiz e parte aérea, confirmando ser uma planta mais mi cotrófica, como comprovado neste trabal ho.

Neste experimento, não foi encontrada correlação significativa entre comprimento de MEA e MET de FMAs em nenhum porta-enxerto utilizado, contrariandoSylvia (1988), indicando não depender da atividade micelial do MET, e vice-versa.

\section{CONCLUSÕES}

1. Há variação no comprimento de micélio extrarradicular ativo e total de fungos micorrízicos arbusculares quando submetidos a diferentes doses 
de $\mathrm{P}$ e porta-enxertos de citros, a qual pode estar ligada ao método empregado de avaliação e, ou, à dependência mi corrízica do hospedeiro na absor ção de nutrientes.

2. As doses crescentes de $P$ proporcionaram dimi nuição da porcentagem de col onização radicular deambos os porta-enxertos eaumentos nas variáveis altura, diâmetro, matéria seca da parte aérea e quantidade total absorvida de macro e micronutrientes por ambos os porta-enxertos.

\section{LITERATURA CITADA}

ABBOTT, L.K. \& ROBSON, A.D. Formation of external hyphae in soil by four species of vesicular-arbuscular mycorrhizal fungi. New Phytol, 99:245-55, 1985.

ABBOTT, L.K.; ROBSON, A.D. \& DE BOER, G. The effect of phosphorus on the formation of hyphae in soil by the vesicular-arbuscular mycorrhizal fungus, Glomus fasciculatum. New Phytol., 97:437-46, 1984.

ANTUNES, V. \& CARDOSO, E.J .B.N. Growth and nutrient status of citrus plants as influenced by mycorrhiza and phosphorus application. Plant Soil, 131:11-19, 1991.

BAGO, B.; AZCÓN-AGUILAR, C.; GOULET, A. \& PICHÉ, Y. Branched absorbing structures (BAS): a feature of the extraradical mycelium of symbiotic arbuscular mycorrhizal fungi. New Phytol., 139:375-388, 1998.

BEEVER, R.E. \& BURNS, D.J.W. Phosphorus uptake, storage and utilization by fungi. In:WOOLHOUSE, H.W. Advances in botanical research. London, Academic Press, 1980. 286p.

BENEFIELD, C.B.; HOWARD, P.J.A. \& HOWARD, D.M. The estimation of dehydrogenase in soil. Soil Biol. Biochem., 9:67-70, 1977.

BETHLENFALVAY, G.J . \& AMES, R.N. Comparation of two methods for quantifying extraradical mycelium of vesiculararbuscular mycorrhizal fungi. Soil Sci. Soc. Am. J ., 51:834837, 1987.

BUWALDA, J.G. \& GOH, K.M. Host-fungus competition for carbon as a cause of growth depressions in vesiculararbuscular mycorrhizal ryegrass. Soil Biol. Biochem., 14:103-106, 1982.

CARDOSO FILHO, J.A. Quantificação do micélio extramatrical de Glomus etunicatum e da sua atividade, em simbiose com milho. Piracicaba, ESALQ/USP, 1994. 121p. (Tese de Mestrado)

CARDOSO, E.J.B.N.; ANTUNES, V.; SILVEIRA, A.P.D. \& OLIVEIRA, M.H.A. Eficiência de fungos micorrízicos vesículo-arbusculares em porta-enxertos de citros. R. Bras. Ci. Solo, 10:25-30, 1986.

COOPER, K.M. \& TINKER, P.B. Translocation and transfer of nutrients in vesicular-arbuscular mycorrhizas - IV. Effect of environmental variables on movement of phosphorus. New Phytol., 88:327-339, 1981.

CRUSH, J.R. E ndomycorrhizas and legume growth in some soils of the Mackenzie Basin, Canterbury, New Zealand. New Zealand J ournal of Agricultural Research, v.19, p.473-476, 1976.
DI BONITO, R.; ELLIOT, M.L.; DES J ARDIN, E.A. Detection of an arbuscular mycorrhizal fungus in roots of different plant species with the PCR. Appl. Environ. Microbiol., 61:28092810, 1995.

FONSECA, E.B.A.; OLIVEIRA, E.; SOUZA, M. \& CARVALHO J.G. Efeitos do fósforo e fungo MVA na nutrição de dois porta-enxertos de citros. Pesq. Agropec. Bras., 29:1889-1896, 1994.

GIOVANETTI, M. \& MOSSE, B. An evaluation of techniques for measuring vesicular arbuscular mycorrhizal infection in roots. New Phytol., 84:489-500, 1980.

GOMES, V.F.F. Desenvolvimento de fungos micorrízicos arbusculares em três espécies de porta-enxertos cítricos sob níveis de fósforo. Piracicaba, ESALQ/USP, 1997. 89p. (Tese de Doutorado)

GRAHAM, J.H.; LEONARD, R.T. \& MENGE, J.A. Membranemediated decrease in root exsudation responsible for phosphorus inhibition of vesicular-arbuscular mycorrhiza formation. Plant Physiol., 68:548-552, 1981.

HAMEL, C.; FYLES, H. \& SMITH, D.L. Measurement of development of endomycorrhizal mycelium using three different vital stains. New Phytol., 115:297-302, 1990.

J AKOBSEN, I.;ABBOTT, L.K. \& ROBSON,A.D. External hyphae of vesicular-arbuscular mycorrhizal fungi associated with Trifolium subterraneum L. 1. Spread of hyphae and phosphorus inflow into roots. New Phytol., 120:371-380, 1992.

J AKOBSEN, I.; J ONER, E.J . \& LARSEN, J. Hyphal phosphorus transport, a keystone to mycorrhizal enhancement of plant growth. In: GIANINAZZI, S. \& SCHÜEPP, H., eds. I mpact of arbuscular mycorrhizas on sustainable agriculture and natural ecosystems. Boston, 1994. p.133-146.

KABIR, Z.; O'HALLORAN, I.P.; FYLES, J.W. \& HAMEL, C. Seasonal changes of arbuscular mycorrhizal fungi as affected by tillage practices and fertilization: Hyphal density and mycorrhizal root colonization. Plant Soil, 192:285-293, 1997.

KABIR, Z.; O'HALLORAN, I.P. \& HAMEL, C. The proliferation of fungal hyphae in soils supporting mycorrhizal and non mycorrhizal plants. Mycorrhiza, 6:477-480, 1996.

KOUGH, J .; MALAJ CZUK, N. \& LINDERMAN, R.G. Use of the indirect immunofluorescent technique to study the vesicular-arbuscular fungus Glomus epigaeum and other Glomus species. New Phytol., 94:57-62, 1983.

LAMBAIS, M.R. \& CARDOSO, E.J.B.N. Avaliação da germinação de esporos de fungos micorrízicos vesículo-arbusculares e da colonização micorrízica de Stylosanthes guianensis em solo ácido e distrófico. R. Bras. Ci. Solo, 12:249-255, 1988.

LAMBAIS, M.R. \& CARDOSO, E.J.B.N. Response of Styl osanthes guianensis to endomycorrhizal fungi inoculation as affected by lime and phosphorus applications. I. Plant growth and development. Plant Soil, 129:283-289, 1990.

LAMBAIS, M.R. \& MEHDY, M.C. Suppression of endochitinase, $\beta$-1,3-endoglucanase, and chalcone isomerase expression in bean vesicular-arbuscular mycorrhizal roots under different soil phosphate conditions. Mol. Plant Microb. Interact., 6:75-83, 1993.

LAMBAIS, M.R. \& MEHDY, M.C. Different expression of defenserelated genes in arbuscular mycorrhiza. Can. J. Bot., 73:533-540, 1995. 
LANFRANCO, L.; WYSS, P.; MARZACHÌ, C. \& BONFANTE, P. DNA probes for identification of the ectomycorrhizal fungus Tuber magnatum Pico. FEMS Micro. Biol. Lett., 114:245252, 1993.

LANFRANCO, L.; WYSS, P.; MARZACHİ, C. \& BONFANTE, P. Generation of RAPD-PCR primers for the identification of isolates of Glomus mosseae, an arbuscular mycorrhizal fungus. Mol. Ecol., 4:61-68, 1995.

MELLONI, R. \& CARDOSO, E.J.B.N. Quantificação de micélio extrarradicular de fungos micorrízicos arbusculares. I. Metodologia empregada. R. Bras. Ci. Solo, 23:53-58, 1998.

MELLONI, R.; NOGUEIRA, M.A.; FREIRE, V.F. \& CARDOSO, E.J.B.N. Avaliação do estado nutricional de limoeiro cravo (Citrus limonia) submetido à doses crescentes de fósforo e espécies defungos micorrízicos arbusculares. In: REUNIÃO BRASILEIRA DE FERTILIDADE DO SOLO E NUTRIÇÃO DE PLANTAS, 22., Manaus, 1996. Anais. Manaus, Sociedade Brasileira de Ciência do Solo, 1996a. p.462-463.

MELLONI, R.; NOGUEIRA, M.A.; FREIRE, V.F. \& CARDOSO, E.J.B.N. E feito de doses de fósforo e fungos micorrízicos arbusculares no crescimento de mudas de limão cravo (Citrus limonia L. Osbeck). In: CONGRESSO LATINO AMERICANO DE CIÊNCIA DO SOLO, 13., Águas de Lindóia, 1996. Trabalhos. Campinas, USP/SLCS/SBCS/ CEA/SBM, 1996b. (CD-ROM)

MILLER, R.M.; J ARSTFER, A.G. \& PILLAI, J.K. Biomass allocation in an Agropyron smithii-Glomus symbiosis. Am. J. Bot., 74:114-122, 1987.

MIRANDA, J.C.C. \& HARRIS, P.J . Effects of soil phosphorus on spore germination and hyphal growth of arbuscular mycorrhizal fungi. New Phytol., 128:103-108, 1994a.

MIRANDA, J.C.C. \& HARRIS, P.J . The effect of soil phosphorus on the external mycelium growth of arbuscular mycorrhizal fungi during the early stages of mycorrhiza formation. Plant Soil, 166:271-280, 1994b.

MIRANDA, J.C.C.; HARRIS, P.J . \& WILD, A. Effects of soil and plant phosphorus concentrations on vesicular-arbuscular mycorrhiza in sorghum plants. New Phytol., 112:405-410, 1989.

NAGAHASHI , G.; DOUDS J r., D.D. \& ABNEY, G.D. Phosphorus amendment inhibits hyphal branching of the VAM fungus Gigaspora margarita directly and indirectly through its effect on root exudation. Mycorrhiza, 6:403-408, 1996.

NOGUEIRA, M.A. Colonização radicular e produção de micélio externo por duas espécies de fungos micorrízicos arbusculares em soja submetida a doses de fósforo. Piracicaba, ESALQ/USP, 1997. 92p. (Tese de Mestrado)

OLIVEIRA, A.A.R. \& J ESUS, I.S. Efeito da infecção por fungos micorrízicos vesicular-arbusculares sobre o desenvolvimento de porta-enxertos de citros. In: CONGRESSO BRASILEIRO DE FRUTICULTURA, 9, Local, 1987. Anais. Local, Editora, 1987. p. 319-325.

PENG, S.; EISSENSTAT, D.M.; GRAHAM, J .H.; WILLIAMS, K. \& HODGE, N.C. Growth depression in mycorrhizal citrus at high-phosphorus supply. Plant Physiol., 101:1063-1071, 1993.

PHILLIPS, J.M. \& HAYMAN, D.S. I mproved procedures for clearing roots and staining parasitic and vesiculararbuscular mycorrhizal fungi for rapid assessment of infection. Trans. Brit. Mycol. Soc. 55:158-160, 1970.
SARRUGE, J.R. \& HAAG, H.P. Análises químicas em plantas. Piracicaba, ESALQ, 1974. 56p.

SCHUBERT, A.; MARZACHÍ, C.; MAZZITELLI, M.; CRAVERO, M.C. \& BONFANTE-FASOLO, P. Development of total and viable extraradical mycelium in the vesicular-arbuscular mycorrhizal fungus Glomus clarum Nicol . \& Schenck. New Phytol., 107:183-190, 1987.

SENA, J .O.A.; STEFANUTTI, R.; MALAVOLTA, E.; CARDOSO, E.J.B.N. \& LABATE, C.A. Cinética de absorção de P tabaco (Nicotiana tabacum) submetido a doses de $P$ e fungos micorrízicos arbusculares. In: CONGRESSO BRASI LEIRO DE CIÊNCIA DO SOLO, 26, Rio de J aneiro. 1997. Anais. Rio de J aneiro, SBCS-CNPS-CNPAB-UFRRJ -PESAGRO, 1997. (CD-ROM)

SILVA, L.R.C.; PEREIRA, J . \& AZEVEDO, I.C. Efeito de fontes de fósforo sobre o crescimento micelial de Gigaspora gigantea in vitro. R. Bras. Ci. Solo, 15:169-72, 1991.

SILVEIRA, A.P.D. \& CARDOSO, E.J.B.N. Kinetics of phosphorus uptake, growth and mineral nutrition of mycorrhizal and non-mycorrhizal bean (Phaseol us vul garis L.). In: NORTH AMERICAN CONFERENCE ON MYCORRHIZAE, 8., Wyoming, 1990. Abstracts. Wyoming, 1990. p.264.

SILVEIRA, A.P.D. Micorrizas. In: CARDOSO, E.J.B.N.; TSAI, S.M. \& NEVES, M.C. eds. Microbiologia do Solo. Campinas, Sociedade Brasileira de Ciência do Solo, 1992. p.257-282.

SIQUEIRA, J.O.; HUBBELL, D.H.; VALLE, R.R. Effects of phosphorus on formation of the vesicular-arbuscular mycorhizal symbiosis. Pesq. Agropec. Bras., 19:1465-1474, 1984.

SOUZA, E.F.O.; SOUZA, M. \& OLIVEIRA, E. E feito de fungos MVA e fósforo sobre o crescimento do limoei ro cravo (Citrus limonia OSBEC"K), pós repicagem. Ci. Prat., 15:398-404, 1991.

SYLVIA, D.M. Activity of external hyphae of vesicular-arbuscular mycorrhizal fungi. Soil Biol. Biochem., 20:39-43, 1988.

SYLVIA, D.M. Quantification of external hyphae of vesiculararbuscular mycorrhizal fungi. Methods in Microbiol., 24:5465, 1992.

THOMSON, B.D.; ROBSON, A.D. \& ABBOTT, L.K. Effects of phosphorus on the formation of mycorrhizas by Gigaspora calospora and Glomus fasciculatum in relation to root carbohydrates. New Phytol., 103:751-765, 1986.

TOBAR, R.; AZCÓN, R. \& BAREA, J.M. I mproved nitrogen uptake and transport from $15 \mathrm{~N}$-labelled nitrate by external hyphae of arbuscular mycorrhiza under water-stressed conditions. New Phytol., 126:119-122, 1994.

VILARIÑO, A.; ARINES, J. \& SCHÜEPP, H. Extraction of vesicular-arbuscular mycorrhizal mycelium from sand samples. Soil Biol. Biochem., 25:99-100, 1993.

WILSON,J .M.;TRINICK, M.J . \& PARKER, C.A.Theidentification of vesicular-arbuscular mycorrhizal fungi using immunofluorescence. Soil Biol. Biochem., 15:439-445, 1983.

ZONTA, E.P.; MACHADO, A.A. \& SILVEIRA J r., P. Sistemas de análise estatística para microcomputadores (SANEST). Pelotas, Universidade Federal de Pelotas,3 1984. 151p. 